\title{
Presencia del titoniano superior en el sur-oeste del lago Titicaca, Perú. implicaciones cartográficas y correlación mundial con base en Himalayites treubi Douville, 1912
}

\author{
C. Esquivel-Macías ${ }^{1 *}$, V. Newton-Machaca², E. Mendoza-Hermosillo ${ }^{3}$ \\ 1 Universidad Autónoma del Estado de Hidalgo. Centro de Investigaciones en Ciencias de la Tierra y \\ Materiales, Colonia Carboneras, C.P.42184, Pachuca de Soto, Hidalgo, México. \\ 2 Universidad del Altiplano-Puno. Facultad de Ingeniería Geológica y Metalúrgica. Jr. Jorge Basadre 640 \\ C.U. Puno, Perú. \\ ${ }^{3}$ Universidad Autónoma del Estado de Hidalgo. Centro de Investigaciones Biológicas, Colonia Carboneras, \\ C.P.42184, Pachuca de Soto, Hidalgo, México.
}

*Autor de Correspondencia: galeon10@yahoo.com

\section{RESUMEN}

Con motivo de la actualización de la geología regional del cuadrángulo de Puno (32v) a escala I: 50,000, se iniciaron en Perú diversas labores de campo, como la de esta contribución que documenta resultados de rocas mesozoicas del Grupo Yura en las inmediaciones del distrito de Tiquillaca, (SW del Lago Titicaca), cuyos afloramientos solían asignarse a rocas Jurásicas del Grupo Lagunillas. El afloramiento se halla en el cerro Yana Apacheta donde se levantó el perfil correspondiente encontrando el amonita Himalayites treubi Douville, 1912; estableciendo correlación con las Fomaciones Chachacumane y Labra. El taxón se determina e interpreta en el área de Ciencias de la Tierra y Materiales (UAEH) con base en cinco ejemplares y en sus parámetros estándar de enrrollamiento y ornamentación. Se considera que el hallazgo de amonitas del Titoniano en el cuadrángulo Puno (32v) amplía y documenta la columna previamente reportada para la localidad y establece clara correlación con sedimentos de Indonesia. Asimismo, se compara la naturaleza litológica y facial de estas rocas con las del Titoniano superior de la Formación Pimienta en México, ya que representan el escaso Titoniano superior que aflora en superficie y un tiempo de intensa reorganización tectónica del continente.

Palabras clave: Amonites, Himalayites, Titoniano, Puno, Perú.

\section{INTRODUCCIÓN}

La presente investigación se desarrolló en el marco del Proyecto GR21 "Geología de los bordes de la Cordillera Oriental y su relación con la zona subandina y altiplano y con los recursos geológicos", de la Dirección de Geología Regional del Instituto Geológico Minero y Metalúrgico (INGEMMET); con la cátedra de geología de Campo II de la Facultad de Ingeniería Geológica y Metalúrgica de la Universidad Nacional del Altiplano (FIGIM-UNAPUNO), con datos propios del proyecto, provenientes de la campaña de campo correspondiente al presente año, próximos a publicarse donde se considera que el hallazgo de amonitas del Titoniano en el cuadrángulo Puno 32v sensu [10] amplía y documenta la columna previamente reportada para la localidad, y con la colaboración 
técnica para la determinación taxonómica y la interpretación ambiental y correlación estratigráfica del Área Académica de Ciencias de la Tierra de la UAEH.

El presente estudio busca caracterizar afloramientos con invertebrados fósiles en el Distrito de Tiquillaca al SO del Lago Titicaca, donde se ha producido históricamente información relevante, sobre evolución y características geológicas con la concurrencia de rocas y fósiles. Informes de campo y tesis de grado a nivel regional han servido como referencia para el presente estudio dentro de los cuales podemos señalar los estudios del proyecto integrado del sur e informes de las cátedras de paleontología, de la fauna amonoidea y se han dado los primeros aportes en la cronología de las capas del Grupo Lagunillas [5].

El objetivo es dar a conocer las correlaciones y el material mencionado para realizar las descripciones taxonómicas; asimismo dar a conocer cómo esta fauna traza correlación mundial con Indonesia en la cuenca del Circumpacífico para contribuir al mejor conocimiento del Grupo Yura y su incorporación dentro de la zonación estratigráfica para el Titoniano [7]; [9].

\section{LOCALIZACIÓN Y GEOLOGÍA REGIONAL.}

Se llega al sitio fosilífero transitando por la carretera Puno-Tiquillaca Km 11. Saliendo de esta última ciudad con rumbo hacia el Sur, a pocos kilómetros de recorrido, se toma la desviación al Cerro Yanaorco. Los afloramientos forman parte de un anticlinal (rumbo del eje S75E), en el cual se encuentran los ejemplares estudiados. Sobre tal trayecto se ha medido la sección, en el afloramiento ubicado con GPS en 370211 y 247373, a un costado del camino (Figura 1).

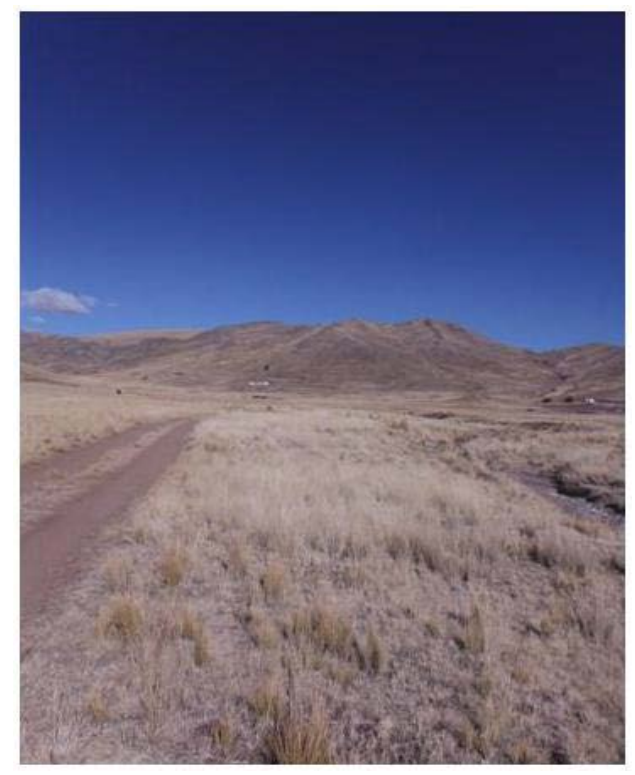

Figura 1. Afloramiento Cerro Yana Apacheta, vista al Oeste

En la cuenca Mesozoica Arequipa las rocas volcánicas del arco Jurásico Chocolate [6]; [8]), infrayacen a sucesiones detríticas y carbonatadas depositadas durante el Jurásico Inferior a Medio, estas unidades han sido denominadas en Arequipa como las 
formaciones Chocolate y Socosani. Sobre estas, se describe al Grupo Yura (Formaciones: Puente, Cachios, Labra y Gramadal) [6]; [3]; [13]; mientras que en Puno, se ha considerado al Grupo Lagunillas [5], como las unidades correlacionables con el Grupo Yura (Formaciones Puente y Cachios). De acuerdo a [13] y con las observaciones aún inéditas del proyecto GR21, la información obtenida en la zona de Tiquillaca, tanto estratigráfica como estructuralmente, tienen una gran similitud con la geología de la zona de Yura, donde las columnas estratigráficas levantadas en el cerro Yanacoto y la quebrada del Burro en Yura están conformadas por conglomerados y areniscas [6]. Este material detrítico, es el producto de la erosión de importantes y potentes zonas volcánicas (Arco Jurásico Chocolate).



Figura 2. Ubicación de las Formaciones y Geología Regional. El Jurásico se representa en tonalidades de color azul, tomado de [10].

\section{MÉTODO}

Se inspeccionó el afloramiento en busca de fósiles posteriormente al periodo de avenidas, lo que facilitó la recolecta de amonites mayores a 5 centímetros, así como fragmentos que se reconstruyeron con soporte de yeso. El muestreo de fósiles y litología macroscópica se realizó conjuntamente con el levantamiento del perfil local del cerro Yana Apacheta. Los amonitas fueron descritos con base en el número de vueltas, diámetro máximo, altura de la vuelta externa y su proporción respecto a la segunda vuelta, así como la tasa de enrollamiento compuesta por los parámetros W, D, S.

\section{RESULTADOS}

Cinco ejemplares de amonoides contenidos en sendas concreciones calcáreas con limolita arenosa; determinados como Himalayites in Uhlig in [4] con sinonimia en la especie $\boldsymbol{H}$. treubi Douville, 1912. Indican el Titoniano Superior.

Tal determinación se otorga con base en: Concha evoluta con vueltas interiores ligeramente comprimidas y vuelta externa ligeramente redondeada en sección transversal. El Costillaje de las vueltas internas es biplicado y simple. Sobre las vueltas medias y externas hay tubérculos esporádicos meso-laterales (espinas) desde las que ramifican 2 a 4 costillas secundarias. Hay costillas primarias intermedias entre las anteriores que 
permanecen simples y costillas secundarias alternas entre las primarias. El vientre muestra un surco medio o una franja plana lisa, que se difumina sobre la última vuelta. Los ejemplares se apegan respecto a la diagnosis anterior, excepto en que las costillas biplicadas de las vueltas internas casi no son visibles. Adicionalmente los parámetros $\mathrm{W}$ (tasa de expansión de la vuelta), D (distancia de apertura del eje de enrollamiento) y $\mathrm{S}$ (forma de la apertura definida por el eje del enrollamiento) que describen la proporción y tasa de enrollamiento son de $1.46,0.450 .61$ respectivamente, lo que corresponde a enrollamiento semi-evoluto basado en las medidas: $a=20.97 \mathrm{~mm}$ de altura de la vuelta; $b=12.88 \mathrm{~mm}$ de espesor de la vuelta; $c=17.00 \mathrm{~mm}$ de radio umbilical mayor; $d=37.61$ $\mathrm{mm}$ de radio mayor; $\mathrm{e}=31.04 \mathrm{~mm}$ de radio menor. Esta determinación hace sinonimia con Himalayites treubi Böhem de la figura 4 a y b de [12] placa 86, misma publicada en [2] pero que se menciona como Himalayites negherburghi Böhem 1904.

Descripción ejemplar 1. Se apega respecto a la diagnosis, excepto que las costillas biplicadas de las vueltas internas casi no son visibles, pero si se aprecian mejor que en la figura $468-5^{a}$ de Arkell [2]. Los tubérculos en realidad son bases de espinas rotas que de todas maneras se insinúan en dicha figura para conformar las del presente ejemplar con una frecuencia poco mayor que en la última vuelta y se ven en esta como si se acercaran más al vientre; pero no es así, porque la naturaleza evoluta de la concha - al sobreponerse a la penúltima vuelta - da esa impresión; ello deja claro que la designación original [2] (sensu Uhlig en [4]) no es exacta y se sugiere que se asigne a semi-evoluta dado que; tanto en la fig. 468-5a como en el presente ejemplar la tasa de enrollamiento es 1.78, que corresponde a semi-evoluta. Debe mencionarse que utilizando la proporción de altura externa de la penúltima vuelta sobre la ultima, en ambos ejemplares, resulta el mismo valor de 0.56 , lo que confirma a $H$. treubi. Adicionalmente los parámetros $\mathrm{W}$ (tasa de expansión de la vuelta), D (distancia de apertura del eje de enrollamiento), S (forma de la apertura definida por el eje del enrollamiento) que describen la proporción y tasa de enrollamiento en este ejemplar son de 1.46, 0.450 .61 respectivamente, lo que corresponde a un enrollamiento semi-evoluto ya que, mientras $\mathrm{D}$ sea mayor respecto a $\mathrm{W}$ la concha será más involuta y viceversa, mientras menor sea $\mathrm{D}$ con respecto a $\mathrm{W}$ la concha será mas evoluta, resultado basado en las medidas: $a=20.97 \mathrm{~mm}$ de altura de la vuelta, $b=12.88 \mathrm{~mm}$ de espesor de la vuelta, $\mathrm{c}=17.00 \mathrm{~mm}$ de radio umbilical mayor, $\mathrm{d}=$ $37.61 \mathrm{~mm}$ de radio mayor, $\mathrm{e}=31.04 \mathrm{~mm}$ de radio menor

Descripción del ejemplar 2. Proporción 0.45 basada en la relación altura externa de la tercera vuelta con respecto a la segunda, ya que la primera está deteriorada. El valor 0.45 es idéntico con la proporción del ejemplar de la figura 468-5a de Arkell [2] y distinto de proporción 0.56 utilizando segunda y primera vuelta, lo que indica que la tasa de enrollamiento aumenta en la última vuelta de ambos ejemplares, rasgo que debería añadirse a la diagnosis original del genero por coincidir con precisión. También se estrechan las vueltas interiores de acuerdo con Uhlig y el comportamiento globoso de la última vuelta, así como la misma ornamentación descrita en el primer ejemplar; pero no se pueden tomar todas las medidas W, D, S, así que sólo se calcula $D=0.59$.

Descripción del ejemplar 3. Amonita de tamaño comparable, sólo se aprecia un segmento del costado, insuficiente para describir la ornamentación, pero sin elementos para negar 
que se trata de $H$. treubi con lo que es la determinación especifica más probable (Himalayites ¿treubi).

Descripción del ejemplar 4. A diferencia del ejemplar 3 se ve un cuarto de vuelta externa, suficiente para ver la base de las espinas del hombro y el la ornamentación trifurcada con algunas costillas primarias y secundarias en el mismo patrón que $H$. treubi, llegando al vientre donde se observa ligeramente aplanado. No se pueden hacer las medidas W, D, $\mathrm{S}$, ni la proporción de altura exterior de las vueltas, no obstante se acepta como $\boldsymbol{c f}$. $\boldsymbol{H}$. treubi dado el contexto de los anteriores.

Descripción del ejemplar 5. Evidencia vaga de costillaje y un fragmento de otro ejemplar adherido. La parte visible sugiere alta tasa de enrollamiento, dado el contexto y la indicación de un surco ventral se sugiere la presencia de Himalayites sp.

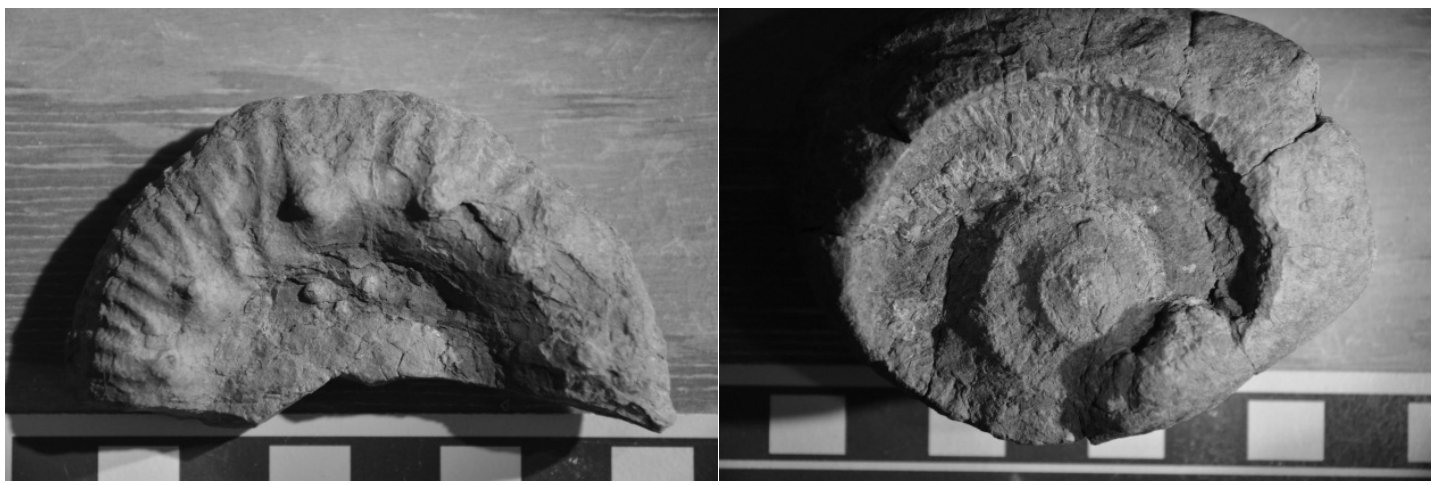

Ejemplar P1

Ejemplar P2

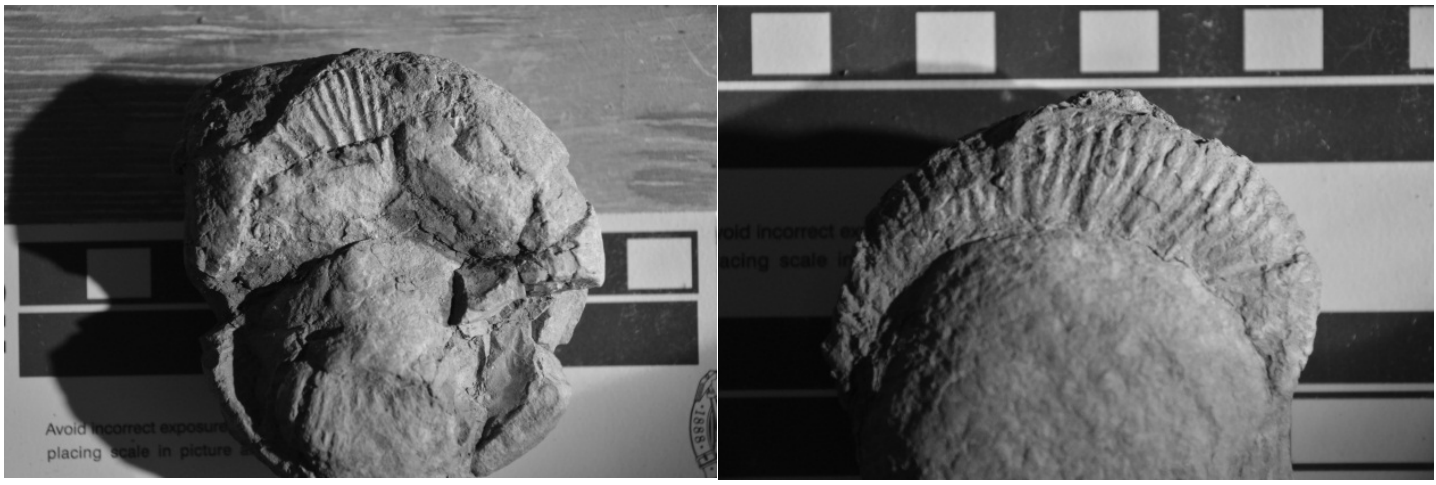

Ejemplar P3

Ejemplar P4 


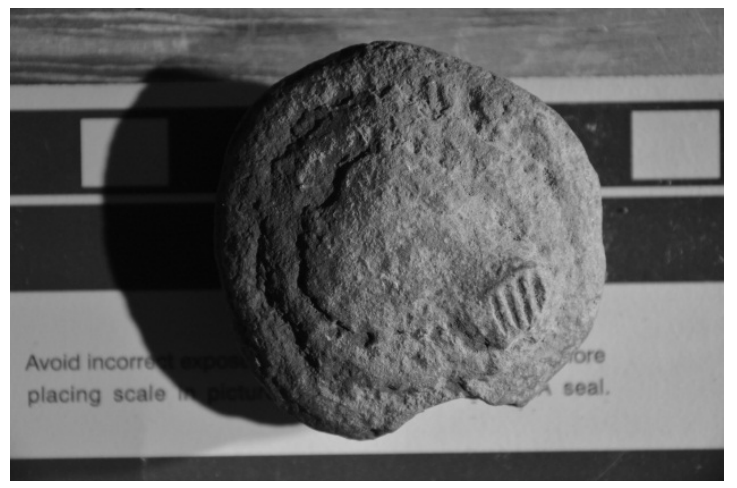

Ejemplar P5

Figura 3. Himalayites treubi

\section{DISCUSIÓN y CONCLUSION}

Esta determinación taxonómica hace sinonimia con el Himalayites treubi Böhem de la figura $4 a$ de Westermann (2000) placa 86, misma publicada en [2] pero mencionada como Himalayites negherburghi Böhem 1904, lo que implica que Uhlig utilizo los trabajos de Böhem [4]. Ambos Himalayites están indicados como índice para el Titoniano Superior de Indonesia, por lo que, dada la continua comunicación transpacífica que ya había desde entonces, podría asumirse la misma edad para la costa de Sudamérica, en particular para Perú.

H. treubi hace clara correlación regional con la Formación Labra en Arequipa y con la Fm. Chachacumene en Tacná. La correlación de Puno con estas formaciones aclara la división con la formación Cachios. Aunque se consideran en la figura 4 como pertenecientes al grupo Yura, es evidente que es una asignación en desuso y hay que señalar que la división bioestratigráfica con la edad Titoniana, marca una diferencia que hace los estratos fosilíferos dejen de ser grupo Yura, si es que la transición estratigráfica/petrológica así lo permiten desde un punto de vista ambiental sedimentario; pero si la litología da indicios de transición sedimentaria gradual o de continuidad entonces podrían ser indicios de que la división (zonación estándar) con el Titoniano pudiera reconsiderarse localmente. Por otra parte la correlación con el Titoniano superior de Indonesia traza correlación cronológica con el Jurásico del circumpacífico.

Por otra parte los pulsos de transgresión/regresión de la parte final del jurásico (figura 4) deberán confirmarse con la litología de esta localidad, los fósiles están en arenisca limosa carbonatada, asociable a fondos neríticos en un contexto de regresión, como los ilustrados en la última fase del Kimeridgiano de la Fm. Cachios (Figura 4): Podría interpretarse que el final del Jurásico dio lugar a grandes cambios de terrestralización de ese sector del continente sudamericano; Es decir que la secuencia Yana Apacheta y su equivalente "Labra" podrían representar la transición hacia el Cretácico.

La determinación taxonómica de los 5 ejemplares de amonoides estudiados complementan la descripción de Jurásico Medio e Inferior realizada por A. Alván \& A. Acosta [1]. Adicionalmente, brindan información sobre las condiciones de regresión que 
inician el Titoniano Superior de México mostrando que son muy semejantes a las que dan lugar a la conformación moderna del continente americano tanto en Sudamérica como en México, las cuales están siendo estudiadas por los autores en la Formación Pimienta del Titoniano mexicano que también representa una cuenca de baja energía con acumulación orgánica en el curso de los eventos anóxicos oceanográficos mundiales sensu [12] y que después fue sustituida transicionalmente por cuencas someras del cretácico que produjeron abundantes carbonatos [11] sin haber transición litoestratigráfica clara; pero al parecer si bioestratigráfica.

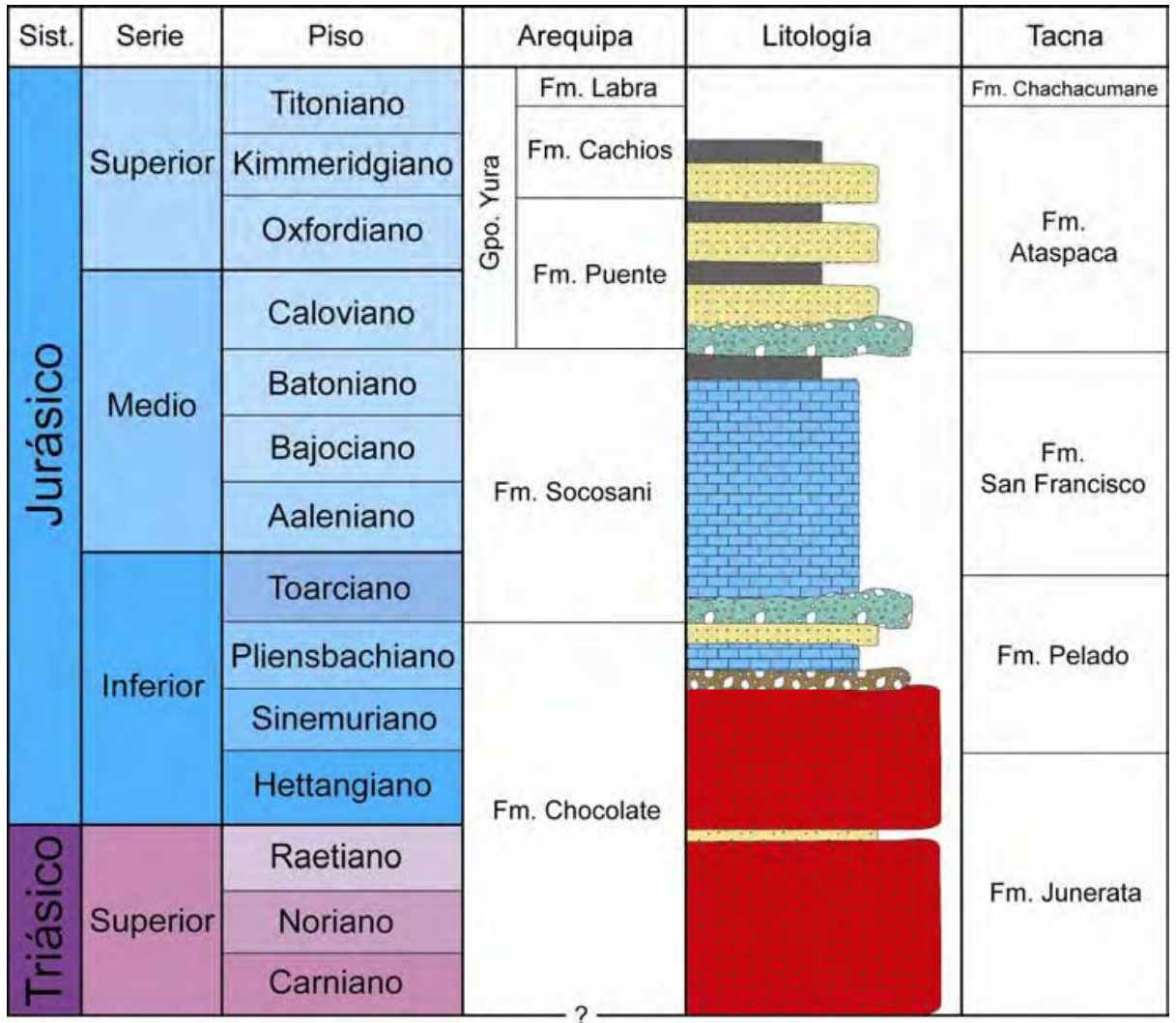

Figura 4. Columna estratigráfica generalizada de los estratos Jurásicos de la cuenca Arequipa, tomado de [13]. Donde aun no se han registrado los sedimentos del Titoniano. Las transgresiones y regresiones del Oxfordiano y Kimeridgiano son evidentes en la quinta columna.

\section{AGRADECIMIENTOS}

Se agradece el apoyo al INGEMMET por su apoyo para el desarrollo de campo de esta investigación, así como a los estudiantes de la Universidad del Altiplano que acompañaron el trabajo de campo y las recolectas; asimismo, se agradece al bachiller Pedro Alejandro Esquivel-León por su ayuda en la medición y determinación de los ejemplares fósiles. Se reconoce especialmente a los Doctores Márius Ramírez-Cardona y Kinardo Flores-Castro quienes establecieron los vínculos académicos entre la UAEH y la Universidad del Altiplano. 


\section{BIBLIOGRAFÍA}

[1]. A. Alván \& A. Acosta. Sedimentary facies and ammonites relations between Arequipa and Tacna during Lower to Middle Jurassic. 21st Lateinamerika Kolloquium LAK 2009. Abstracts (2009) 72-77.

[2]. W. Arkell, W. Furnish, B. Klummel, A. Miller, R. Moore, O. Schindewolf, P. SylvesterBradley and C. W. Wrigth. Treatise on invertebrate paleontology. Part. L. Mollusca 4 Cephalopoda Ammonoidea. Geological Society Of America and University Of Kansas Press (1957) 490.

[3]. V. Benavidez. Estratigrafía Pre-terciaría de la Región de Arequipa. II Congreso Nacional de Geología, Boletín de la Sociedad Geologica del Perú. 38 (1962) 5-63.

[4]. G. Böhem. Die Sudkusten der sula-instein Taliabu and Magnoli. 1. Grenzchiten zswichen Jura und Kreide (1904). Palentogr. Suppl. 4 (1904-12) 1-179.

[5]. E. Jaillard, H. Capeta, M. Ellenberg, M. Feist, N. Grambfest-Fossand, J. Lefernc and B. Sigg. Sedimentology, Palaeontology, Biostratigraphy and Correlation of the Cretaceous Vilcheca Group of southern Peru. Cretaceous Research. 19 (1973) 623661.

[6]. W. Jenks. La geología de la región Arequipa y sus alrededores. Información y Memorias de la sociedad de Ingenieros del Perú. (1948) 104.

[7]. [7] I. León. Antecedentes sedimentológicos del Jurásico-Cretácico inferior en la zona de Yura. Tesis de Bachiller, Universidad Nacional de San Agustín (1981) 100.

[8]. W. Martínez y J. Cervantes. Rocas Ígneas en el sur del Perú. Nuevos datos geocronométricos, geoquímicos y estructurales de los paralelos $16^{\circ}$ y $18^{\circ} 30^{\prime}$ Latitud sur. Bol 26/ serie D. (2003) 146.

[9]. A. Pino, T. Semperé, J. Jacay \& M. Fornari. Estratigrafía, paleogeografía y paleo tectónica del intervalo Paleozoico superior-Cretáceo inferior en el área de Mal PasoPalca (Tacna). Publicación Especial No 5, Sociedad Geológica del Perú (2004) 1544.

[10]. L. Vargas. Geología del cuadrángulo de Arequipa (Hoja 33-s). Servicio de Geología y Minería de Perú actualmente INGEMMET. Serie A. Carta Geológica Nacional 24 (1970) 64-78.

[11]. G. Westermann. Ammonite Bio-Chronology and Biogeography of the Circum-Pacific Middle-Jurassic Systematics Association. Special Volume 18 (1980) 459-498.

[12]. S. Westermann, K. B. Föllmi, T. Adatte, V. Matera, J. Schnyder, D. Fleitmann, N. Fiet, I. Ploch, S. Duchamp-Alphonse. The Valanginian $\delta^{13} \mathrm{C}$ excursion may not be an expression of a global oceanic anoxic event, Earth and Planetary Science Letters 290 (2010) 118-131.

[13]. J. C. Vicente. Elementos de la Estratigrafía Mesozoica sur-peruana. Comité sudamericano del Jurásico y Cretácico. Cuencas sedimentarias del Jurásico de América del Sur Vol. 1 (1981) 319-331. 